\title{
Review of: "Renal function and lipid metabolism are major predictors of circumpapillary retinal nerve fiber layer thickness-the LIFE-Adult Study"
}

Eun Ji Lee

Potential competing interests: The author(s) declared that no potential competing interests exist.

In a large population-based dataset, Rauscher et al showed that decreased renal function and abnormal lipid metabolism may be respectively associated with thinner and thicker circumpapillary retinal nerve fiber layer (cpRNFL) thicknesses as assessed by OCT.

It is an interesting and well-performed study with one major limitation that retinopathies (diabetic or hypertensive retinopathy, retinal vein occlusion, etc.) were not considered as an exclusion criteria. Retinal pathology (edema, hemorrhage, etc.) can significantly influence the measured cpRNFL, so it is not clear whether the increased/decreased systemic parameters themselves, or the retinal pathology caused by the systemic condition affected the cpRNFL thickness measurement.

The study meticulously discussed the relationship between the abnormal lipid profile and increased cpRNFL thickness, while lacks how the decreased renal function was associated with thinner cpRNFL. As the authors mention in the discussion section, it is reasonable to assume that brain lesion should be associated with cpRNFL thickness. However, I wonder how the cpRNFL thickness could be influenced by the renal function.

Lastly, each systemic factor was differentially associated with the cpRNFL thickness in different sectors. It would be interesting to know how such differential relationships existed for the varying systemic parameters. 